\title{
Development a quantitative method for the determination of misoprostol acid in human serum
}

\begin{abstract}
Summary
There has been conducted an experimental study and longitudinal design with which method highly sensitive and specific has been developed, using the equipment ultraperformance liquid chromatograph with a detector mass - mass (UPLC - MS - MS) for the qualitative and quantitative determination of ester misoprostol through its metabolite misoprostol acid in serum samples. A peak was obtained at $2.2 \mathrm{~min}$ of chromatographic running and the results are between 3.1 and 18,4ppb.
\end{abstract}

Keywords: acid misoprostol, serum samples, liquid chromatography, metabolite, solid phase extraction

\author{
Volume 7 Issue 5 - 2019 \\ Luis Alberto López Avila \\ Laboratory of Toxicology, Institute of Legal Medicine, Peru \\ Correspondence: Luis Alberto López Avila, Public Ministry, \\ Institute of Legal Medicine, Laboratory of Toxicology, Av. Juan \\ Vicente Nicolini 256, Urb.Palao-SMP, Peru, \\ Email luisalbertolopeza@gmail.com
}

Received: September 24, 2019 | Published: October 25, 2019

\section{Introduction}

The present work aims to develop a qualitative quantitative method using the UPLC MS-MS equipment, for the determination of misoprostol ester through its metabolite, misoprostol acid, in human serum samples who consumed misoprostol ester for abortive purposes. This study was conducted in the Toxicology Laboratory of the Institute of Legal Medicine of the Public Ministry. At the national level there is no official method and internationally there are methods in similar teams1 The highly sensitive and specific qualiquantitative method of the UPLC MS-MS team, which is developed in the present study, can determine misoprostol ester through its serum acid misoprostol metabolite of pregnant women who have ingested misoprostol ester in abortive doses.

\section{Material and methods}

Types of study: The study is experimental and longitudinal in design.

\section{Samples and pre-treatment}

Methods were tested using liquid-liquid extraction with various pure organic solvents and mixtures, which gave non-reproducible results. Techniques were then tested using solid phase extraction cartridges (SPE) for the pre-treatment of biological samples. LC18 , LC-8 and hydrophilic-lipophilic balance, cationic and anion exchange (HLB) cartridges that were investigated using Oasis ${ }^{\circledR}$ protocols and those recommended by each manufacturer were tested; each of $3 \mathrm{~mL}$ and $30 \mathrm{mg}$ with various dilutions, conditions, washing and circumvention reagents. HLB Oasis ${ }^{\circledR}$ cartridges gave consistent results in terms of recovery of misoprostol acid in addition to obtaining a cleaner target. SPE HLB cartridges were preconditioned with one $\mathrm{mL}$ of methanol solution: water $(1: 1)$, then the sample was loaded. Water-methanol solution (9:1) was used as the wash solution, then the analyte was eluted with a $\mathrm{mL}$ of pure methanol to which one milliliter of water was added, placing the mixture in a $1.5 \mathrm{~mL}$ vial from which it was injected only five $\mu \mathrm{L}$ in the LC-MS / MS.

\section{Sampling (Sampling)}

Frozen blood or serum samples were received, taken at the different headquarters of the Institute of Legal Medicine nationwide.
After centrifuging the samples and separating the serum, they were accepted for analysis.

\section{Method Development}

\section{Liquid chromatography}

To obtain a symmetrical peak and a retention time of $\sim 2.2 \mathrm{~min}$ (Figure 1), several solvent mixtures such as water and methanol were tested using different proportions, with average flows of 0.3-0.6 mL/ min, giving better results the mixture of methanol: water (Table 1).

\section{Mass spectrophotometry}

To optimize the negative electro spray ionization (ESI) conditions for misoprostol acid, quadrupole scans were performed in negative ion mode. During a direct infusion experiment, the spectral mass for misoprostol acid revealed a peak at $\mathrm{m} / \mathrm{z} 367.1 \mathrm{amu}$. When experimented with misoprostol acid $\mathrm{m} / \mathrm{z} 367.1$ amu in MRM mode, the collision energy originated a son ion of $\mathrm{m} / \mathrm{z} 249.0 \mathrm{amu}$.

\section{Selectivity}

Control human serum (analyte free) and human serum inoculated with misoprostol acid were injected whose samples underwent the SPE process described above. No interference peak of endogenous compounds was observed at the retention time of the analyte sample compared to the white sample. The retention time of the misoprostol acid was $2.2 \mathrm{~min}$. The chromatographic run time was five min (Figure 1).

\section{Calibration curve}

The calibration curve was constructed using calibration standards of $1 ; 3 ; 5 ; 10 ; 15$ and $20 \mathrm{ppb}$. The calibration curve was prepared by determining the best average peak response - area vs concentration and adequate at $\mathrm{y}=\mathrm{mx}+\mathrm{c}$.

\section{Sample Preparation}

The blood or serum samples collected belonging to cases of possible abortions were processed as follows: 1 . Five $\mathrm{mL}$ of blood sample was obtained and poured into a glass tube, then centrifuged, giving one $\mathrm{mL}$ of serum; This milliliter was subjected to solid phase 
extraction with a three $\mathrm{mL}$ HLB cartridge, preconditioned with a $\mathrm{mL}$ of water solution: methanol (1:1), washed with a $\mathrm{mL}$ of water: methanol solution (9: 1) and the analyte eluted with one $\mathrm{mL}$ of methanol, one $\mathrm{mL}$ of water was added at the end and five $\mu \mathrm{L}$ was injected into the LC-MS / MS system.

\section{Identification and quantification of misoprostol in serum}

An LC system equipped with an isopump degasser with autosampler was used to inject five $\mu \mathrm{L}$ aliquots of the processed samples; The system had an Acquity UPLC BEH C18 column (1.7 $\mu \mathrm{m} ; 2.1$ X 50 $\mathrm{mm})$, maintained at room temperature $\left(24 \pm 2^{\circ} \mathrm{C}\right)$. The mobile gradient phase, with an electrospray ionization mass spectrophotometer (ESI), is presented in Table 1 .

Table I Gradient mobile phase used in the MS-MS LC

\begin{tabular}{llll}
\hline Weather & $\begin{array}{l}\text { Flow } \\
\text { (mL/min) }\end{array}$ & $\begin{array}{l}\text { \% A } \\
\text { (Water) }\end{array}$ & $\begin{array}{l}\text { \%B } \\
\text { (methanol) }\end{array}$ \\
\hline & 0,4 & 95 & 5 \\
3 & 0,4 & 5 & 95 \\
3,5 & 0,4 & 5 & 95 \\
3,6 & 0,4 & 95 & 5 \\
5,5 & 0,4 & 95 & 5
\end{tabular}

Quantification was performed by MS-MS detection in negative ion mode (ESI negative) for misoprostol acid. The cone voltage was $25 \mathrm{~V}$ and collision gas was $20 \mathrm{~V}$. Ion detection was performed in the multiple reaction monitoring mode (MRM), the transition pairs of misoprostol acid m/z $367.1 \mathrm{amu}$ and, as a son ion (daughter ion) $\mathrm{m} / \mathrm{z} 263.0 \mathrm{amu}$. The analytical data were processed by the statistical program SPSS v19

Description of statistical methods that were used to analyze the results
a) Pearson's correlation
b) Average concentration
c) Coefficient of variance
d) Comparison of means

\section{Standard solutions}

The primary stock solution $1.00 \mathrm{mg} / \mathrm{mL}$ of misoprostol acid was prepared in a mixture of water: methanol (1:1), liquid chromatography grade and stored at $-4^{\circ} \mathrm{C}$. Appropriate dilutions were made with methanol: water mixture $(1: 1)$, to which working stock solutions of $1 ; 3 ; 5 ; 10$ and $20 \mathrm{ppb}$ on the day of analysis and these solutions were used to prepare the curve. The work solutions in duplicate were stored at approximately $-4^{\circ} \mathrm{C}$ for one week, and each one was read three times.

\section{Results}

The results were the following:

Table 2 \& Figure 1

Table 3 shows that there is a positive and significant high correlation $(0.99, \mathrm{p}=0.00<0.05)$ between concentration of misoprostol acid and the area under the curve (Figure 2).

Table 4 shows that the coefficients of variation for the average concentrations are less than $15 \%$ (Table 5, Figure $3 \& 4$ ).

Table 2 Readings obtained with the calibration curve

\begin{tabular}{llllll}
\hline & $\begin{array}{l}\text { Readings } \\
\text { (ppb) }\end{array}$ & Areas & & $\begin{array}{l}\text { Readings } \\
\text { (ppb) }\end{array}$ & Areas \\
\hline 1 & 3,3 & 43,15 & 11 & 3,7 & 49,268 \\
2 & 4,2 & 57,615 & 12 & 13,9 & 198,51 \\
3 & 5,1 & 69,145 & 13 & 7,8 & 106,23 \\
4 & 3,1 & 40,872 & 14 & 16,2 & 231,456 \\
5 & 15,6 & 223,828 & 15 & 11,5 & 163,147 \\
6 & 4,1 & 55,42 & 16 & 18,4 & 265,364 \\
7 & 3,5 & 47,125 & 17 & 12,4 & 176,617 \\
8 & 6,1 & 85,967 & 18 & 8,7 & 122,457 \\
9 & 17,5 & 250,165 & 19 & 13,9 & 197,92 \\
10 & 18,3 & 261,134 & 20 & 15,3 & 220,138 \\
\hline
\end{tabular}

Table 3 Pearson's correlation between concentration of acid misoprostol and the area under the curve

\begin{tabular}{lll}
\hline & & Areas \\
\hline Misoprostol Acid Concentration & Pearson correlation & $0.99(* *)$ \\
& sig. (bilateral) & 0 \\
\hline
\end{tabular}

** The correlation is significant at the 0.0 I level (bilateral).

Table 4 Average concentrations and variance coefficient found for the calibration curve

\begin{tabular}{llllll}
\hline Concentration & ppb & Area & Average ppb & s & $\%$ CV \\
\hline$I$ & $\mathrm{I}$ & 9,512 & & & \\
$\mathrm{I}$ & $\mathrm{I}$ & 9,235 & & & \\
$\mathrm{I}$ & 0,9 & 8,712 & & & \\
$\mathrm{I}$ & $\mathrm{I}, \mathrm{I}$ & 10,869 & 0,966666667 & 0,08164966 & 8,44651635 \\
$\mathrm{I}$ & 0,9 & 8,417 & & & \\
$\mathrm{I}$ & 0,9 & 8,369 & & & \\
3 & 3,2 & 41,667 & & & \\
3 & 2,9 & 36,736 & & & \\
3 & 3,2 & 41,117 & 3,116666667 & 0,11690452 & 3,75094715 \\
3 & 3,2 & 41,625 & & & \\
3 & 3,1 & 40,075 & & & \\
3 & 3,1 & 40,502 & & & \\
\hline
\end{tabular}




\begin{tabular}{|c|c|c|c|c|c|}
\hline Concentration & ppb & Area & Average ppb & s & $\% \mathbf{C V}$ \\
\hline 5 & 5,1 & 68,93 & \multirow{6}{*}{4.983333333} & \multirow{6}{*}{$0.147 \mid 9601$} & \multirow{6}{*}{2.95376618} \\
\hline 5 & 5,2 & 69,714 & & & \\
\hline 5 & 4,9 & 65,737 & & & \\
\hline 5 & 5 & 67,436 & & & \\
\hline 5 & 4,8 & 65,348 & & & \\
\hline 5 & 4,9 & 65,917 & & & \\
\hline 10 & 9,8 & 136,633 & \multirow{6}{*}{9,933333333} & \multirow{6}{*}{0,15055453} & \multirow{6}{*}{ I,5I564964 } \\
\hline 10 & 9,9 & 137,884 & & & \\
\hline 10 & 9,9 & 138,766 & & & \\
\hline 10 & 9,8 & 136,926 & & & \\
\hline 10 & 10,2 & 142,664 & & & \\
\hline 10 & 10 & 139,488 & & & \\
\hline 20 & 20,1 & 286,647 & \multirow{6}{*}{20} & \multirow{6}{*}{0,16733201} & \multirow{6}{*}{0,83666003} \\
\hline 20 & 19,8 & 281,633 & & & \\
\hline 20 & 20,2 & 288,025 & & & \\
\hline 20 & 20,1 & 286,072 & & & \\
\hline 20 & 19,8 & 282,415 & & & \\
\hline 20 & 20 & 285,418 & & & \\
\hline
\end{tabular}

Table 5 Comparison of means of the area under the curve and the concentration of acid misoprostol

\begin{tabular}{|c|c|c|c|c|c|c|c|c|c|}
\hline & & & & ANOVA & & rey test & & & \\
\hline & & & Media & Standard deviation & I & 3 & 5 & 10 & 20 \\
\hline \multirow[t]{5}{*}{ Area down the curve } & one & 6 & 9,2 & 0,9 & & $0,00 *$ & $0,00^{*}$ & $0,00 *$ & $0,00 *$ \\
\hline & three & 6 & 40,3 & $\mathrm{I}, 8$ & & & $0,00 *$ & $0,00 *$ & $0,00 *$ \\
\hline & five & 6 & 67,2 & $0,00 *$ & & & & $0,00 *$ & $0,00 *$ \\
\hline & ten & 6 & 138,7 & 2,2 & & & & & $0,00 *$ \\
\hline & twenty & 6 & 285 & 2,5 & & & & & \\
\hline \multirow[t]{5}{*}{ Misoprostol Acid Concentration } & one & 6 & I & $0, I$ & & $0,00 *$ & $0,00^{*}$ & $0,00^{*}$ & $0,00 *$ \\
\hline & three & 6 & 3,1 & 0,1 & & & $0,00 *$ & $0,00 *$ & $0,00^{*}$ \\
\hline & five & 6 & 5 & $0,00 *$ & & & & $0,00 *$ & $0,00 *$ \\
\hline & ten & 6 & 9,9 & 0,2 & & & & & $0,00 *$ \\
\hline & twenty & 6 & 20 & 0,2 & & & & & \\
\hline
\end{tabular}

* $\mathrm{p}<0.05$ significant

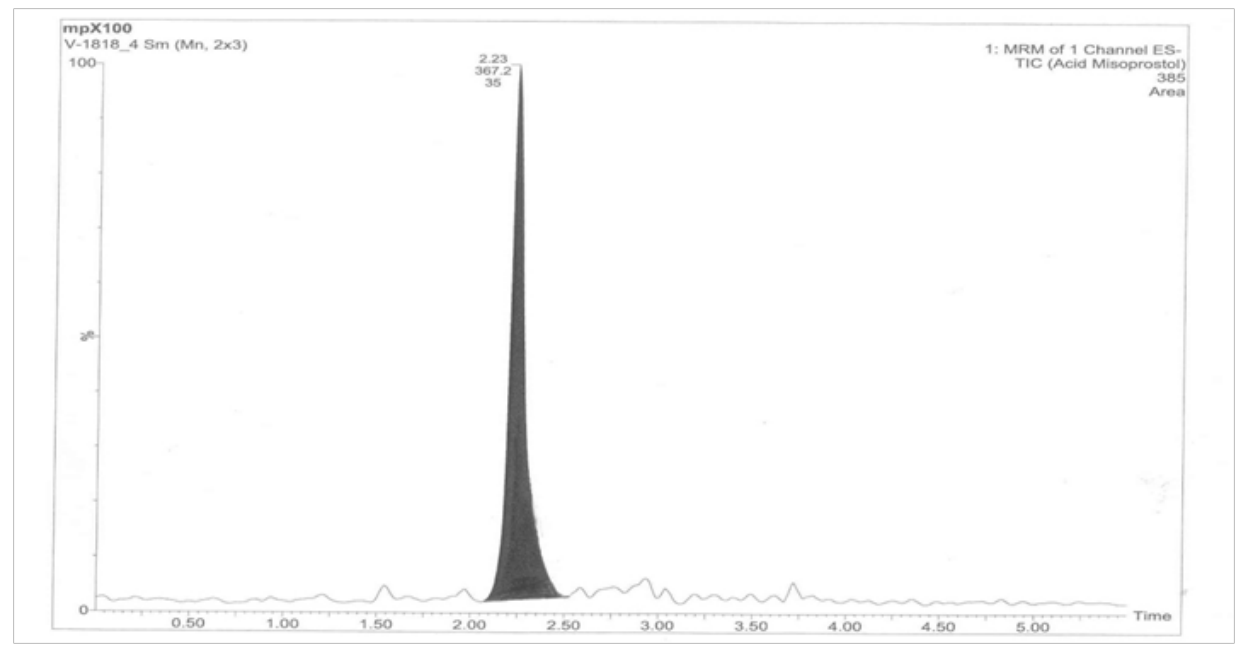

Figure I Chromatogram of Misoprostol acid. 




Figure 2 Concentration in ppb of misoprostol acid and area under the curve.

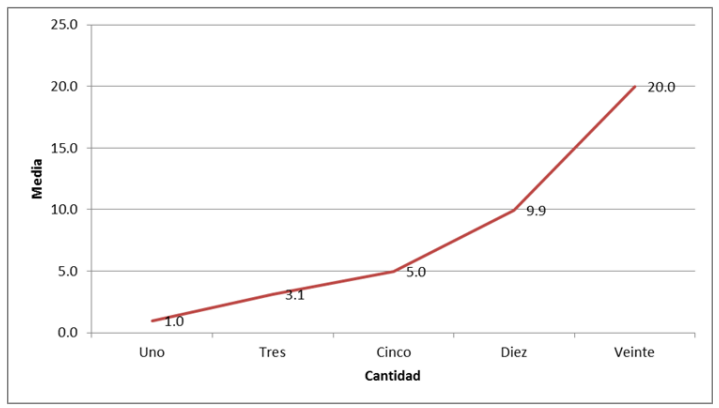

Figure 3 Graph of concentration lines of misoprostol acid.



Figure 4 Area under the acid misoprostol curve.

\section{Discussion}

The dose for antacid therapy purposes is one oral misoprostol tablet $(200 \mu \mathrm{g})$, whose intake results in lower plasma levels than those found in this study; on the other hand, for abortive purposes, three tablets $(600 \mu \mathrm{g})$ oral vial and two tablets vaginally $(400 \mu \mathrm{g})$ are used, making a total of $1000 \mu \mathrm{g}$. An LC-MS/MS method has been developed for the determination of misoprostol acid, using a solid phase extraction (SPE) procedure from serum samples. The curve obtained has concentrations of $1 ; 3 ; 5 ; 10$ and $20 \mathrm{ppb}(\mathrm{x} 10-3 \mu \mathrm{g} / \mathrm{mL})$ in methanol medium: water. Table 2 shows that the values obtained from the samples are within the limits of the curve. According to the bibliographic reference1 the values obtained are in the range of pg/ $\mathrm{mL}$. The main differences between the method of Zou et al., ${ }^{1}$ and the method developed in the present work are the following: a) The dose was $0.6 \mathrm{mg}$, however in our method it was $1 \mathrm{mg}(03$ tablets orally and 02 tablets vaginally)

b) The samples were blood with heparin, this is plasma, instead we use serum.

c) The samples were obtained from a controlled universe, 20 healthy individuals, however in the present study it is an uncontrolled universe.

In the study by Zou et al. , $20 \mu \mathrm{L}$ aliquots were injected into the chromatograph, instead in the present study they were $5 \mu \mathrm{L}$ aliquots. The equipment used by Zou and it was a hybrid LC-MS-MS unit with Agilent brand quaternary pump and Applied Biosystem brand detector, however in this work it was a UPLC MS-MS brand Waters Acquity model. Blood samples, in the study by Zou et al., Were taken at the times of: 7.5; fifteen; 30; Four. Five; 60; 90; 120; 180; 240 and 360 minutes after the dose, however in the present study it is not possible to determine the times in which the samples were taken due to impossibilities of origin such as ignorance of the time of taking the abortive tablets by the pregnant woman. Concentration values in the study by Zou et al. were average values in $\mathrm{pg} / \mathrm{mL}$ at each of the times indicated in 6.6. In contrast, in the present study it was a shot with an unknown time reading, these values are between 3.1 to $18.4 \mathrm{ppb}$.

It is presented as an alternative to traditional liquid-liquid extraction, an extractive method by SPE using the HLB Oasis $\mathrm{Max}^{2}$ $300 \mathrm{mg} \times 3 \mathrm{~mL}$ cartridge, with which the analyte was extracted, eluting with methanol to which water is added giving a methanol mixture: water $(1: 1)$ ready to be injected into the LC system. Table 5 shows that as the amount of acid misoprostol increases, the mean area under the curve increases, statistically significant

Table 5 shows that as the amount of misoprostol acid increases, the mean misoprostol concentration increases, statistically significant. Table 4 shows that the coefficients of variation for the average concentrations of misoprostol acid are less than $15 \%$. According to a bibliographic reference, the plasma concentration of misoprostol ester reaches peaks after 20 minutes of intake. ${ }^{3}$ Unchanged misoprostol (ester) cannot be detected in plasma even five minutes after oral dose. ${ }^{1}$

\section{Conclusion}

a. A qualitative quantitative method was developed for the determination of misoprostol acid using human serum.

b. Misoprostol ester could not be determined in serum samples since misoprostol ester is rapidly metabolized to misoprostol acid, its active metabolite.

c. In the cases received and analyzed the concentrations were determined and quantified, whose values are in the range of 3.1 to $18.4 \mathrm{ppb}$.

d. The concentrations found in our study, which are in the range of 3.1 and $18.4 \mathrm{ppb}$ could not be correlated with the values found in item 1, of bibliographic references, because there are the following differences: types of samples, sampling times, equipment used, population of samples, doses and volumes of aliquots injected into the equipment.

\section{Acknowledgments}

None. 


\section{Conflicts of interest}

Author declares that there is no conflict of interest.

\section{References}

1. Zou Y, Chen X, Song B, et al. Determination of misoprostol acid in human plasma by liquid chromatography coupled to tandem mass spectrometry. J Chromatogr B Analyt Technol Biomed Life Sci. 2007;852(I2): I 22-1 27.
2. Vijaya D, Jagadeesh B, Kumar K, et al. Development and validation of highly sensitive method for determination of misoprostol free acid in human plasma by liquid chromatography electrospray ionization tandem mass spectrometry: Application to a clinical pharmacokinetic study. J Chromatogr B Analyt Technol Biomed Life Sci. 20I I;879(26):2827-2833.

3. Bentov Y, Sheiner E, Katz M. Misoprostol overdose during the first trimester of pregnancy. Eur J Obstet Gynecol Reprod Biol. 2004; I I 5 ( I): I08109. 